\title{
Effect of microstructure changes on magnetic properties of spark plasma sintered Nd-Fe-B powders
}

\author{
W. Kaszuwara ${ }^{1}$, M. Leonowicz ${ }^{1}$, B. Michalski ${ }^{1}$ and M. Lis ${ }^{2}$ \\ ${ }^{1}$ Faculty of Materials Science and Engineering, Woloska 141, 02-507 Warszawa, Poland \\ ${ }^{2}$ Institute of Non-Ferrous Metals, Sowinskiego 5, 44-100 Gliwice, Poland
}

\begin{abstract}
In this study the SPS method was applied for low RE content (8,5\% at.) and high RE content $(13,5 \%$ at.) MQ powders. The powders were sintered in a wide range of temperature, for 5 min., under pressure of $35 \mathrm{MPa}$. The low RE content grade, densified reluctantly and gained the density close to the theoretical value only for $850{ }^{\circ} \mathrm{C}$. The coercivity decreased gradually with increasing sintering temperature. On the other hand, the densification of the higher RE content grade powder occurred much easier and the coercivity, close to the theoretical value, was achieved already at $650{ }^{\circ} \mathrm{C}$. The coercivity of this material also decreased with increasing sintering temperature. Microstructural studies revealed that the SPS sintering process leads to partial decomposition of the $\mathrm{Nd}_{2} \mathrm{Fe}_{14} \mathrm{~B}$ phase. The proportion of the RE-rich and iron phases increases parallel to the increasing sintering temperature. On the basis of the current results one can conclude that fabrication of high density MQ powders based magnets by the SPS method is possible, however the powders having higher RE content should be used for this purpose and the sintering temperature as low as possible, related to density, should be kept.
\end{abstract}

\section{Introduction}

The Nd-Fe-B materials sintered by the spark plasma sintering (SPS) method have already been described in many publications. Most often the materials to be sintered were powders with a micrometric grain size prepared from ingots by crushing and milling and then subjected to sintering. The chemical compositions of the powders were typical of those used in sintered magnets, i.e. the content of the rare earth elements exceeded the stoichiometric $\mathrm{Nd}$ content of the $\mathrm{Nd}_{2} \mathrm{Fe}_{14} \mathrm{~B}$ phase [1-3]. The sintering temperatures were substantially lower than those used in conventional sintering processes, but the best results were achieved at temperatures above $800^{\circ} \mathrm{C}$ $[2,3]$. The density of the sinters increased with increasing sintering temperature, whereas the coercivity and remanence initially increased and, after reaching a maximum, decreased [3]. In the experiments described in refs [1] and [3] the magnetic properties of the sinters produced by the SPS method did not differ essentially from those of the materials sintered by conventional methods.

It has been shown that sintering of the Nd-Fe-B powder by the SPS method affects the grain size in the particles $[4,5]$. A selective growth of the grains, located close to the surface of the particles, was observed. The range, of the resulting coarse grain zone, grows with increasing the sintering temperature. The internal part of the particles is built of the fine grain zone. This finding clarifies, generally observed fact, that sintering of coarser powders provides higher coercivity [6].

The authors of refs [2] and [3] have also found that the magnetic properties of the SPS-sintered materials could be improved by subjecting them to additional heat treatment such as annealing at a temperature of $1000^{\circ} \mathrm{C}$.

When analyzing the microstructure of the Nd-Fe-B sinters, many authors noticed that the sinters contained an Nd-rich phase which formed large precipitates mainly located at the triple junctions between the grains of the $\mathrm{RE}_{2} \mathrm{Fe}_{14} \mathrm{~B}$ phase [1]. The authors of ref. [3] found that during the additional annealing at $1000^{\circ} \mathrm{C}$ the $\mathrm{Nd}$-rich phase forms a new thin layer that isolates the $\mathrm{Nd}_{2} \mathrm{Fe}_{14} \mathrm{~B}$ grains and thereby improves the magnetic properties of the material. Another feature, characteristic of the microstructure of the magnets sintered by SPS, is considerable grain refinement, when compared with conventional sintering.

SPS-sintering also improves the mechanical properties of the material - the authors of ref. [1] achieved an increase of the bending strength by about $40 \%$ compared to that obtained in the materials sintered by conventional methods.

Much room has been devoted in the literature to corrosion examinations of the sinters produced by SPS $[2,7]$. It has appeared that their corrosion resistance is not worse than that of conventionally sintered magnets and, 
in certain environments, it can even be substantially better.

There are also literature reports describing the use of the SPS method for sintering of nanocrystalline Nd-Fe-B powders. These reports are mainly concerned with producing bulk pieces intended for the manufacturing of anisotropic magnets by die-upset-forging [8, 9]. The required density of the nanocrystalline $\mathrm{Nd}-\mathrm{Fe}-\mathrm{B}$ powders was achieved at low process temperatures, even as low as $650^{\circ} \mathrm{C}[8]$.

The SPS method was also used for simultaneous crystallization and densification when pressing amorphous powders prepared from melt-spun ribbons. In this way it is possible to produce Nd-Fe-B composites in the bulk form [10].

In the present study the SPS method was used for sintering of two commercial nanocrystalline ribbon powders, with various $\mathrm{RE}(\mathrm{Nd}$ or $\mathrm{Pr}$ ) contents, designed to be bonded with a plastic material or to be hot-pressed. The aim was to examine the effect of the RE content on the phenomena which proceed in the microstructure of the material and determine the effectiveness of the SPS sintering process and the magnetic properties of the sinters obtained.

\section{Experimental}

Two powders used in the experiments (provided by the Magnequench) differed in their rare earth element contents and magnetic properties. One powder, further denoted as LCP, which contained small amount of rare earth element $(8.5 \mathrm{at} \%$ of $\mathrm{Pr})$ and increased content of boron $(20 \mathrm{at} \%)$, showed a relatively low coercivity, and a high remanence. It is a nanocomposite powder intended for the fabrication of magnets bonded with a plastic material. The other powder, further denoted as HCP, contained a higher content of rare earth elements (about $13.5 \mathrm{at} \%$ of $\mathrm{Nd}$ ) and had a relatively low remanence and a very high coercivity. This powder has been designed to be consolidated by hot pressing.

The powders were subjected to sintering in a graphite die using the SPS method under a pressure of $30 \mathrm{MPa}$. The sintering processes were conducted within a wide temperature range, namely at 650, 700, 750, 800, and $850^{\circ} \mathrm{C}$. In most of the experiments, the sintering time was $5 \mathrm{~min}$, and the heating rate was $250^{\circ} \mathrm{C} / \mathrm{min}$. which is the heating rate close to the maximum capacity of the sintering unit employed and permits the electric pulses to achieve high power. Another experiment included processing of the sinters produced of the HCP powder at $650^{\circ} \mathrm{C}$ at a heating rate of $50^{\circ} \mathrm{C}$ in an $\mathrm{Ar}$ protective atmosphere. These sinters were not subjected to further annealing.

The magnetic properties of the sinters were examined using a VSM magnetometer, and their structure was analyzed by SEM (Zeiss).

\section{Results}

Our experiments have shown that the density of the SPSsintered material depends on the sintering temperature, but this relationship varies depending on the RE element content (Fig.1). The HCP powder, with a higher RE content, is very well consolidated irrespective of the sintering temperature (within the temperature range examined). After the SPS, its density is close to the theoretical value, except after sintering at the highest temperature $\left(850^{\circ} \mathrm{C}\right)$ when it is markedly lower. The effectiveness of the consolidation of the LCP powder, which has a smaller RE content, increases with increasing sintering temperature, but even after sintering at $850^{\circ} \mathrm{C}$, its density is lower by more than $5 \%$ than that of the sintered HCP powder. The theoretical densities of the two powders are similar: for the LCP powder the theoretical value was determined to be $7.690 \mathrm{~g} / \mathrm{cm}^{3}$, and for the $\mathrm{HCP}$ powder - to be $7.676 \mathrm{~g} / \mathrm{cm}^{3}$.

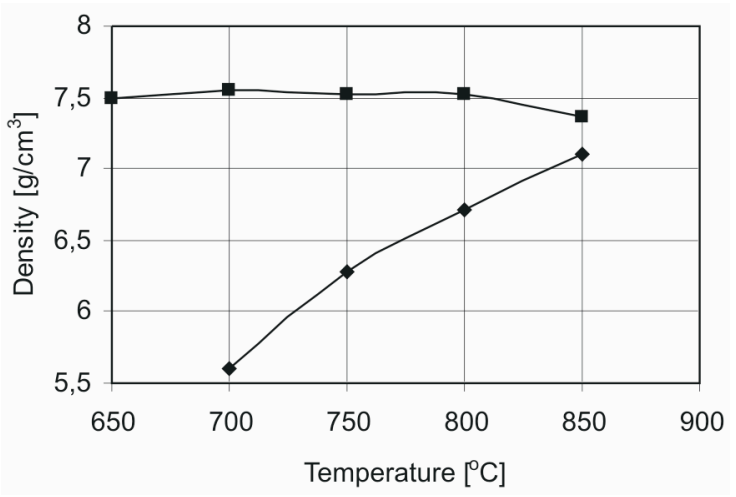

Fig. 1. Effect of the sintering temperature on the density of the sinters produced of the LCP powder $(\downarrow)$ and HCP powder $(\bullet)$.

The dependence of the magnetic properties of the two sintered powders on the sintering temperature is shown in Fig. 2. For both the sinters the coercivity decreases with increasing sintering temperature within the entire temperature range examined. The remanence of the LCP powder initially increases with temperature, which is due to the increasing density of the material, but above $750^{\circ} \mathrm{C}$ it decreases. The remanence of the HCP powder decreases within the entire sintering temperature range. The changes are related to the changes in the phase composition.

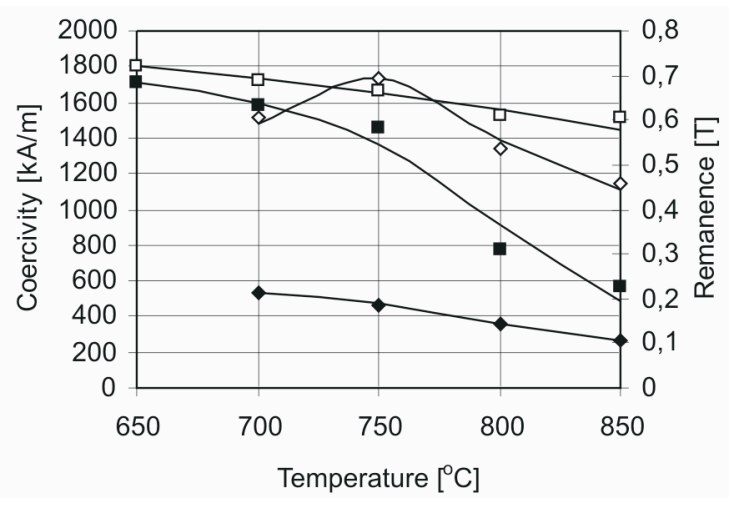

Fig. 2. Effect of the sintering temperature on the magnetic properties. LCP coercivity $(\diamond)$, remanence $(\diamond)$; HCP coercivity $(\square)$, remanence $(\square)$.

Figure 3 shows SEM BSE images of the microstructure of the sintered LCP powder. We can see 
pores and, which is especially interesting, the boundaries between the flake-shaped grains of the powder in the initial state (Fig.3a). At a greater magnification (Fig.3b) precipitates of some other phase are visible between the powder flakes within the boundary regions $1-2 \mu \mathrm{m}$ in diameter. The EDS examinations of the chemical composition of the sintered powder have shown that these precipitates contain $35 \pm 2 \mathrm{at} \%$ of $\mathrm{B}$, whereas the RE (Pr) content is smaller $(6.5 \pm 0.5 \mathrm{at} \%)$ than the average content of this element in the sample $(7.5 \mathrm{at} \%)$.
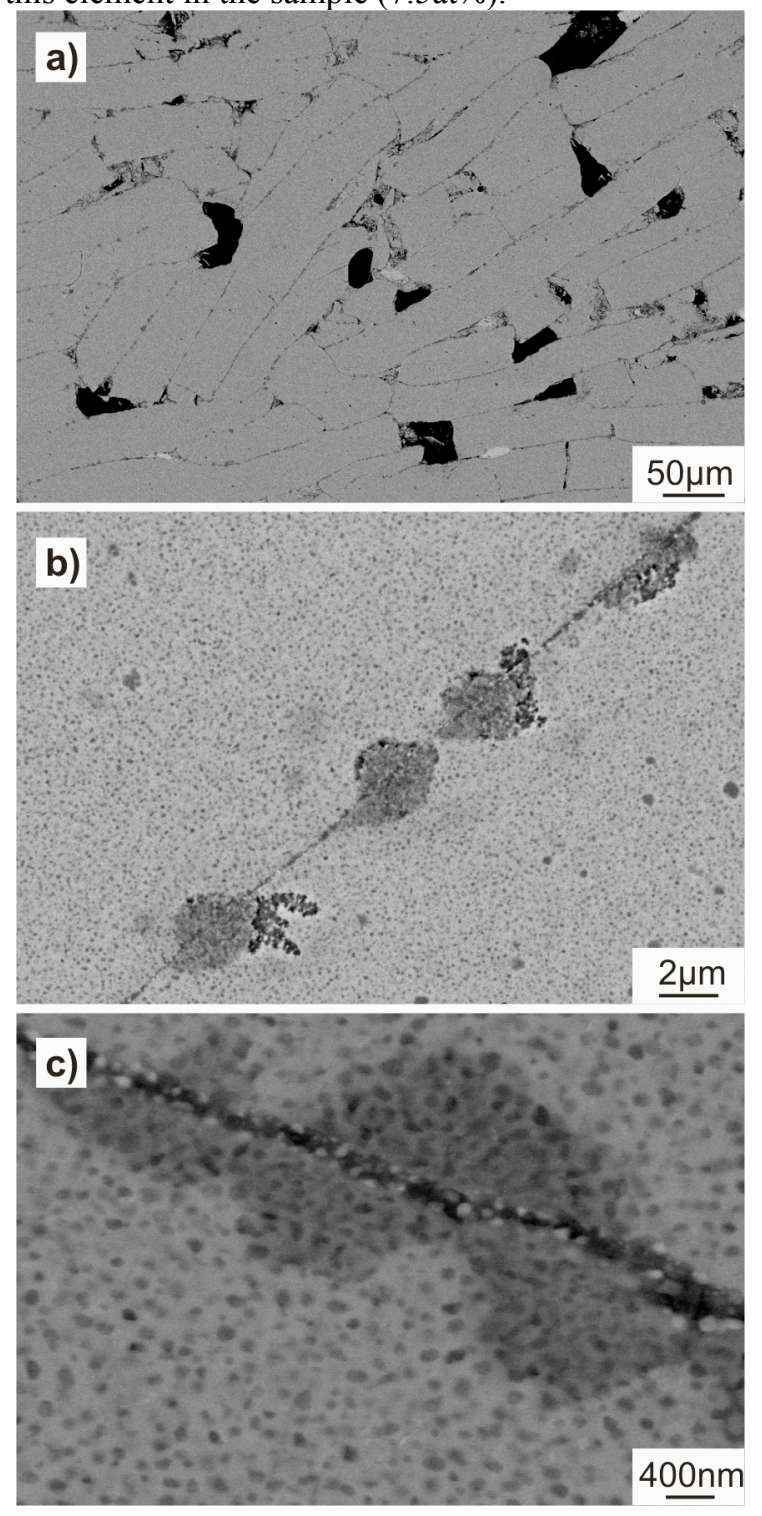

Fig. 3. Microstructure of the LCP powder sintered at a temperature of $750^{\circ} \mathrm{C}$.

Some precipitates have dendrites which have grown into the interior of the grains. They probably have formed at places where the electric discharges, ignited during the SPS process, melted locally the material. The SEM studies, performed at high magnifications in the BSE contrast (Fig.3c), showed that the material contains very small precipitates, in a matrix of the hard magnetic phase, which can be identified as iron borides. They are also visible in the area of flake's interface. In the latter case, however, in the matrix, the other phase from the Pr-Fe-B system, which contains more light elements is present.
Unfortunately, the experimental technique used did not enable complete identification of this phase, one can however assume that this phase is formed in the course of decomposition (melting and crystallization) of the $\mathrm{Pr}_{2} \mathrm{Fe}_{14} \mathrm{~B}$ phase. Its presence proves that, in the process of SPS of low Pr content of Pr-Fe-B powders, local decomposition of the hard magnetic phase occurs. This process affects both the coercivity and remanence of the magnets.

The HCP powder sintered at a temperature of $750^{\circ} \mathrm{C}$ is very well consolidated (Fig.4). The grain boundaries visible in the Figure $4 \mathrm{a}$ are light in color and surrounded with darker regions.

At the higher magnification (Fig.4b) we can see regions, about $100 \mathrm{~nm}$ in size, which are precipitates enriched with $\mathrm{Nd}$ and surrounded with irregular regions enriched with Fe. Examinations of the chemical composition have shown that the light-color regions contain about $24 \mathrm{at} \%$ of $\mathrm{Nd}$, whereas in the darker regions, around the grains, the $\mathrm{Nd}$ content is about $12.5 \pm 0.4 \mathrm{at} \%$ (still farther from the grain boundaries there are light-gray regions, unchanged during the sintering, in which the $\mathrm{Nd}$ content is about $13.5 \mathrm{at} \%$ ). Along the grain boundaries we can also see pores with sizes below $1 \mu \mathrm{m}$. It can be supposed that, in the regions between the powder particles, the Nd-rich phase located on the boundaries of the $\mathrm{Nd}_{2} \mathrm{Fe}_{14} \mathrm{~B}$ grains was melted and displaced (pushed out) into the regions between the
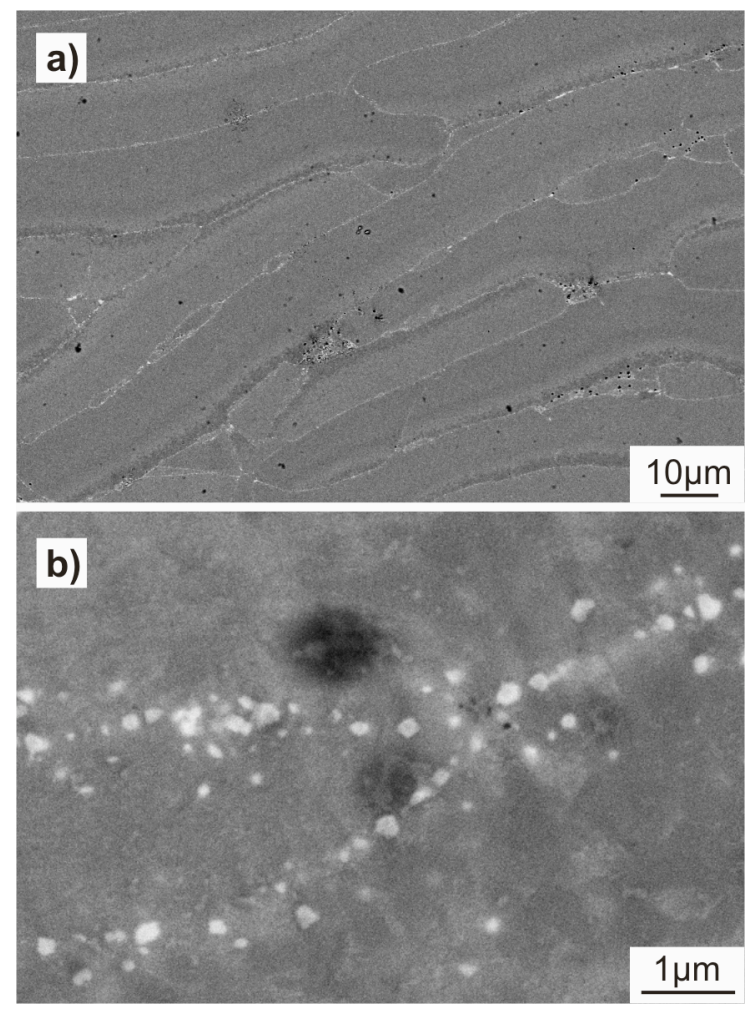

Fig. 4. Microstructure of the HCP powder sintered at a temperature of $750^{\circ} \mathrm{C}$.

boundaries of the powder particles). The content of the isolating phase is reduced so much that the $\mathrm{Nd}_{2} \mathrm{Fe}_{14} \mathrm{~B}$ grains are probably in direct contact with one another. This is the reason why the coercivity of the material decreases. 
The higher the sintering temperature, the greater is the proportion of the regions with the affected microstructure. After sintering at $850^{\circ} \mathrm{C}$ the share of these regions already exceeds $50 \%$ of the material volume.

In the next experiment, the HCP and LCP samples were subjected to SPS-sintering at a temperature of $850^{\circ} \mathrm{C}$ and then to annealing at a temperature of $700^{\circ} \mathrm{C}$ for $30 \mathrm{~min}$. In the LCP samples the coercivity remained almost unchanged whereas the remanence increased by $35 \%$. In the HCP samples, on the other hand, the coercivity increased by $2 \%$ and the remanence decreased by $20 \%$. The difference in the behavior of the two sintered samples during the annealing results from the difference between the microstructural changes that occur in the two samples during the sintering process. In the LCP sample the products into which the $\mathrm{RE}_{2} \mathrm{Fe}_{14} \mathrm{~B}$ phase decomposes during the sintering form a new phase during the annealing (resulting in increased remanence). In the HCP sample the removal of the Nd-rich phase from the regions between the $\mathrm{RE}_{2} \mathrm{Fe}_{14} \mathrm{~B}$ grains during sintering is irreversible at this annealing temperature.

Another observation arising from the microstructure examinations is that the two powders differ in their ability to consolidate. The LCP powder particles, with the content of the rare earth element lower than their stoichiometric content in the $\mathrm{RE}_{2} \mathrm{Fe}_{14} \mathrm{~B}$ phase, do not undergo accommodation i.e. they do not adapt their shape to the surrounding grains. We can see in Fig. 3a that their shapes do not change, but when pressed by the neighboring particles they just break. The grains of the HCP powder, with the RE content greater than the stoichiometric content in the $\mathrm{RE}_{2} \mathrm{Fe}_{14} \mathrm{~B}$ phase, behave in a quite different manner. They deform so as to adapt their shape to the surroundings. This ability to accommodate is associated with the presence of a liquid phase between the nanometric $\mathrm{RE}_{2} \mathrm{Fe}_{14} \mathrm{~B}$ phase grains during the sintering, which permits the powder particles to change their positions with respect to one another. In the micrometric scale, this results in an effect similar to plastic deformation of the powder particles and appreciably facilitates their consolidation.

\section{Summary}

Two RE-Fe-B ribbon powders, differing in their RE contents $(\mathrm{Nd}$ or $\mathrm{Pr})$ and magnetic properties, were subjected to sintering by the SPS method. Both the powders contained the $\mathrm{RE}_{2} \mathrm{Fe}_{14} \mathrm{~B}$ phase. The microstructure and magnetic properties of the SPSsintered powders appeared to depend on the RE content. If the RE content exceeds its stoichiometric content in the $\mathrm{RE}_{2} \mathrm{Fe}_{14} \mathrm{~B}$ phase, the Nd-rich phase is locally melted during the sintering process and forced into the free spaces between the powder particles. The higher the sintering temperature, the larger is the zone around the powder particles in which the phase structure undergoes changes. At the same time the participation of the liquid phase in the sintering process ensures that the powder is well consolidated since the particles can change their shape to fit with the surrounding grains. In the powder with the RE content lower than the stoichiometric content in the $\mathrm{RE}_{2} \mathrm{Fe}_{14} \mathrm{~B}$ phase, it is this phase is partially melted and, in consequence, decomposed into the phases from the Fe-B system. These powders to be consolidated need high sintering temperatures, but even at these temperatures the powder particles do not accommodate their shapes to the neighboring grains. We can conclude that the SPS sintered powders have good magnetic properties, but, because of the unavoidable changes of their microstructure during the sintering process, their magnetic properties are not as good as the properties of the starting powders.

\section{Acknowledgment}

The study was supported by the project co-funded by the European Union in Operational Programme Innovative Economy POIG 01.01.102.00/10 (ZAMAT). The powders were provided by Magnaquench.

\section{References}

1. M. Yue, A.L. Cao, G.P. Wang, W. Q. Liu, D.T. Zhang, J. X. Zhang, Y. P. Luo, X. Y. Zhang, Y. M. $\mathrm{Li}$, Proceedings of 19th International Workshop on Rare Earth Permanent magnets \& Their Applications, 312 (2006)

2. M. Yue, J. Zhang, Y. Xiao, G. Wang, T. Li, IEEE Transaction on Magnetics, 6, 3551 (2003).

3. W. Mo, L. Zhang, A. Shan, L. Cao, J. Wu, M. Komuro, Intermetallics, 15, 1483 (2007).

4. Z. W. Liu, H. Y. Huang, X. X. Gao, H. Y. Yu, X. C. Zhong, J. Zhu, D. C. Zeng, J. Phys. D, Appl. Phys., 44, 025003 (2011)

5. Y. L. Huang, Z. W. Liu, X. C. Zhong, H. Y. Yu, D. C. Zeng, Powder Metallurgy, 55,124-129 (2012).

6. Z. W. Liu, Y. L. Huang, H. Y. Huang, X. C. Zhong, H. Y. Yu, D. C. Zeng, Key Engineering Materials, 510-511, 307-314 (2012).

7. M. Yue, M. Tian, J. X. Zhang, D. T. Zhang, P. L. Niu, F. Yang, Materials Science and Engineering B, 131, 18 (2006).

8. Z. H. Hu, J. Li, L. H. Chu, Y. Liu, Journal of Magnetism and Magnetic Materiale, 323, 104 (2011).

9. M. Yue, L. Zhang, W. Liu, G. Wang, IEEE Transaction on Magnetics, 10, 3892 (2005).

10. K. Tanaka, T. Fukuzaki, K. Nishio, R. Tamura, Journal of Physics: Conference Series, 191, 012025 (2009). 